\title{
Transverse impedances and collective instabilities in a heavy ion accelerator
}

\author{
J. Liu, ${ }^{1,2,3}$ J. C. Yang,,$^{1,3,}$ J. W. Xia, ${ }^{1,3}$ D. Y. Yin, ${ }^{1,3}$ G. D. Shen, ${ }^{1,3}$ P. Li, ${ }^{1,3}$ B. Wu, ${ }^{1,2,3}$ S. Ruan, ${ }^{1,2,3}$ \\ H. Zhao, ${ }^{1,2,3}$ G. Wang, ${ }^{1,2,3}$ Z. Q. Dong, ${ }^{1,2,3}$ K. D. Wang, ${ }^{1,2,3}$ and L. P. Yao ${ }^{1,2,3}$ \\ ${ }^{1}$ Institute of Modern Physics, Chinese Academy of Sciences, Lanzhou, 730000, China \\ ${ }^{2}$ University of Chinese Academy of Sciences, Beijing, 100049, China \\ ${ }^{3}$ Huizhou Research Center of Ion Sciences, Huizhou, 516003, China
}

(Received 21 December 2017; published 12 June 2018)

\begin{abstract}
Evaluation of transverse impedances and collective instabilities is important for determining whether a transverse feedback system or damping schemes should be prepared in the BRing (Booster Ring) at the HIAF (High Intensity Heavy-ion Accelerator Facility). In this paper, some dominant transverse impedances are estimated to build a transverse impedance model of the BRing. With this model, all potential transverse instabilities and their growth times or rates are analyzed by analytical methods or simulations, and the results agree with each other. The growth times of some instabilities are shorter than the duration times of corresponding manipulations, which shows transverse instabilities may have many detrimental impacts on the BRing. To cure the transverse instabilities, a transverse feedback system will be proposed in the design of the BRing. Besides, this paper not only shows the transverse instabilities in the BRing, but also provides the whole method for estimating them in the design of a new accelerator facility.
\end{abstract}

DOI: 10.1103/PhysRevAccelBeams.21.064403

\section{INTRODUCTION}

BRing (Booster Ring) is the main accelerator that accumulates and accelerates high intensity beams with a wide range of heavy ion species in the HIAF (High Intensity Heavy-ion Accelerator Facility) project which is proposed and designed by IMP (Institute of Modern Physics, Chinese Academy of Sciences) [1]. A version of the HIAF design is shown in the Fig. 1. In this design, the BRing has a three-fold and mirror-symmetric lattice over its circumference of $530.8 \mathrm{~m}$ and its maximal momentum rigidity is about $34 \mathrm{~T} \mathrm{~m}$. The main parameters of the BRing for two modes and three reference beams are summarized in the Table I. The whole operating process of the BRing is very complicated. The beam from iLinac (Ion Linac) is accumulated into a coasting beam with uniform distribution in two transverse phase spaces by the two-plane painting injection [2]. This coasting beam is captured into several bunches and accelerated in the first acceleration. At the end of the first acceleration, the beam is unbunched into a coasting beam again. The energy of the beam remains unchanged between two accelerations. Then, the beam is

\footnotetext{
*Corresponding author. yangjch@impcas.ac.cn

Published by the American Physical Society under the terms of the Creative Commons Attribution 4.0 International license. Further distribution of this work must maintain attribution to the author(s) and the published article's title, journal citation, and DOI.
}

captured into single bunch and accelerated to the maximal energy in the second acceleration [3]. Finally, a coasting beam is used in the slow extraction.

Transverse collective instabilities stimulated by transverse impedances can have many detrimental impacts on the beam in the high intensity accelerators. The beam may suffer distortion, displacement or emittance growth, which results in the beam loss $[4,5]$. Evaluation of transverse impedances

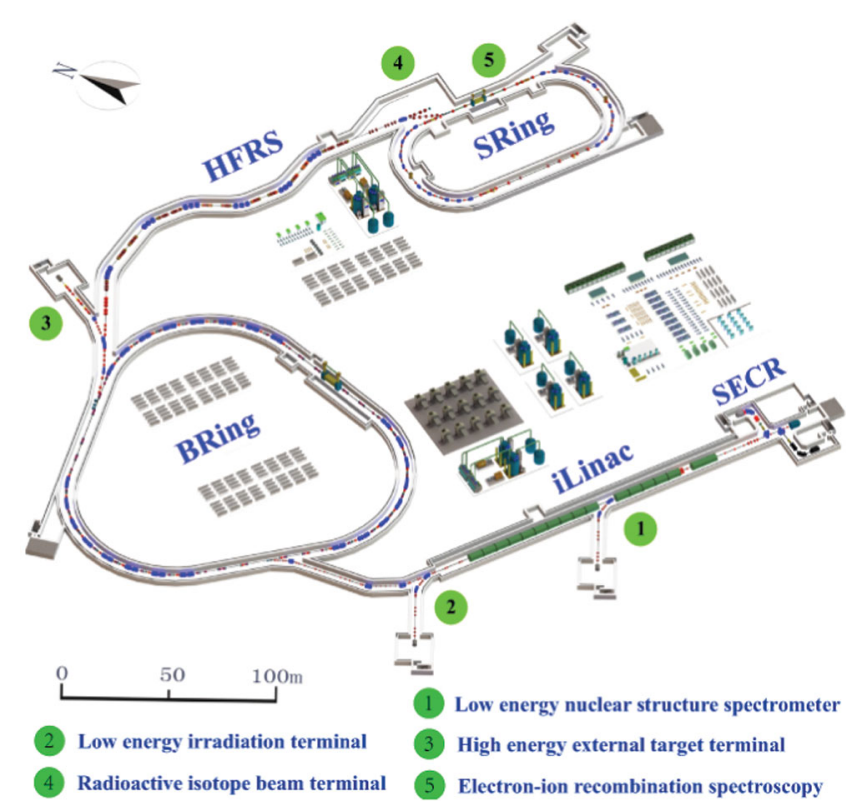

FIG. 1. The layout of the HIAF project. 
TABLE I. The main parameters of the BRing.

\begin{tabular}{|c|c|c|}
\hline Parameter & Proton mode & Heavy ion mode \\
\hline Circumference $C$ & \multicolumn{2}{|c|}{$530.8 \mathrm{~m}$} \\
\hline Transition energy $\gamma_{t}$ & 11.21 & 7.64 \\
\hline Transverse tune $\nu_{H} / \nu_{V}$ & $11.45 / 8.43$ & $8.45 / 7.43$ \\
\hline Average $\beta$ function $\beta_{H} / \beta_{V}$ & $7.4 / 10.0 \mathrm{~m}$ & $13.4 / 14.3 \mathrm{~m}$ \\
\hline Natural chromaticity $\xi_{H} / \xi_{V}$ & $-18.34 /-12.45$ & $-9.53 /-9.58$ \\
\hline Acceptance $\varepsilon_{H} / \varepsilon_{V}$ & $200 / 100 \pi \mathrm{mm} \mathrm{mrad}$ & $200 / 100 \pi \mathrm{mm} \mathrm{mrad}$ \\
\hline Injection momentum spread $\delta\left(3 \delta_{r m s}\right)$ & \pm 0.002 & \pm 0.002 \\
\hline Required intensity $N_{\text {beam }}$ & $2 \times 10^{12} \mathrm{ppp}(\mathrm{p})$ & $\begin{array}{l}3 \times 10^{11} \operatorname{ppp}\left(\mathrm{Kr}^{19+}\right) \\
1 \times 10^{11} \operatorname{ppp}\left(\mathrm{U}^{34+}\right)\end{array}$ \\
\hline Number of bunches (first/second acceleration) & $2 / 1$ & $3 / 1$ \\
\hline Kinetic energy $E_{k}$ (injection/middle/extraction) & 48/1000/9300 MeV/u (p) & $\begin{array}{c}30 / 400 / 1750 \mathrm{MeV} / \mathrm{u}\left(\mathrm{Kr}^{19+}\right) \\
17 / 200 / 800 \mathrm{MeV} / \mathrm{u}\left(\mathrm{U}^{34+}\right)\end{array}$ \\
\hline
\end{tabular}

and collective instabilities is very significant for analyzing whether the BRing can reach the required intensity in the Table I and obtain high quality beam without extra stablization. And only after this evaluation, it can be determined if some components for stabilization, including transverse feedback system and other damping sources like octupole magnets, should be introduced [6,7]. This must be finished in the design because the components will occupy the space and cause costs. Therefore, the estimates of transverse impedance model and collective instabilities are inevitable in the design of the BRing or other high intensity accelerators.

In this paper, some dominant impedance sources in the BRing, mainly resistive wall and kickers, are considered and estimated, which leads to a BRing transverse impedance model. Using this impedance model, transverse modecoupling instability, transverse coupled-bunch instability and transverse unbunched beam instability are analyzed by analytical methods or CISP (Simulation Platform for Collective Instabilities) simulations [8]. The results given by different methods agree with each other. Their growth times or rates are compared with the duration times of corresponding manipulations to decide whether the transverse instabilities can influence the BRing. However, in some more realistic conditions, the analytical models are no longer applicable. For these situations, some simulations are added in this paper to further make sure that it is appropriate to apply these results into the design. Now, the comparisons and results show that the BRing may suffer some of three instabilities in the future, and a transverse feedback system should be designed and added into the BRing. Besides, this paper also aims at providing the whole general method to analyze transverse impedances and collective instabilities in the design of a new high intensity accelerator.

\section{TRANSVERSE COUPLING IMPEDANCES IN THE BRING}

The dominant transverse impedance sources in the BRing mainly consist of resistive wall and extraction kickers. The transverse resistive wall impedance and transverse kicker impedance both have a resistive part and can influence the growth rates directly. The estimates of these two transverse impedances have been preliminarily finished in the BRing and the results have been used in the analysis of all the transverse instabilities. Some transverse broadband impedances from bellows, holes, steps, collimators, etc. are also calculated. These transverse impedances only have imaginary part and just contribute to the short-range wake. A BRing transverse impedance model is given by these estimates.

\section{A. Transverse resistive wall impedance}

The imperfectly conducting chamber can introduce the transverse resistive wall impedance [4]. And there is no good way to reduce this impedance without increasing the costs much. The vacuum chamber of the BRing is made of 316L stainless steel. In order to avoid the eddy current in the first acceleration, the vacuum chamber thickness is around $0.3 \mathrm{~mm}$ [9]. The transverse resistive wall impedance is also related to the size of the vacuum chamber and should be integrated over the ring.

In the calculation of the transverse resistive wall impedance, it is very important to choose the specific model according to whether the skin depth at the lowest frequency is smaller than the vacuum chamber thickness. The transverse resistive wall impedance can influence the beam at the lowest frequency $\omega=(1-[Q]) \omega_{0}$, where $[Q]$ is the fractional part of the betatron tune and $\omega_{0}$ is the revolution angular frequency. It is known as $(1-Q)$ line [5]. The $\mathrm{U}^{34+}$ beam with the injection energy has the lowest frequency $\omega=0.368 \mathrm{MHz}$ in the horizontal direction. Then the maximal skin depth in the stainless-steel chamber of the BRing is $0.237 \mathrm{~mm}$ and this maximal depth is less than the thickness of the vacuum chamber. It is appropriate to apply the classical formula in the whole frequency range while calculating and using the transverse resistive wall impedance in the BRing.

After simplifying racetrack-like chambers into rectangular chambers, the transverse resistive wall impedance of the BRing is estimated by the classical formula as [4] 


$$
\begin{aligned}
& Z_{1, r w}^{H}(\omega)=\frac{1.9 \times 10^{5}}{\sqrt{|\omega|}}(\operatorname{sign}(\omega)-i)(\mathrm{k} \Omega / \mathrm{m}), \\
& Z_{1, r w}^{V}(\omega)=\frac{5.6 \times 10^{5}}{\sqrt{|\omega|}}(\operatorname{sign}(\omega)-i)(\mathrm{k} \Omega / \mathrm{m}),
\end{aligned}
$$

where $\omega$ is the angular frequency, $Z_{1, r w}^{H}$ is the horizontal resistive wall impedance, $Z_{1, r w}^{V}$ is the vertical resistive wall impedance and sign is the sign function. The difference between the horizontal impedance and the vertical impedance mainly comes from the rectangular chambers. And the resistive part of the transverse resistive wall impedance behaves as $Z_{1, r w}^{\perp} \propto \operatorname{sign}(\omega) \times|\omega|^{-1 / 2}$, which is very important.

\section{B. Transverse kicker impedance}

In the BRing, seven kicker modules with the windowframe magnets are used for the fast extraction. For each module, the main parameters are shown in the Table II. It is very difficult to get the accurate transverse kicker impedance without bench measurements [10]. The generator and the cable that are connected to the kicker magnet construct a circuit with the magnet, which introduces significant difference between the total impedance and the magnet impedance. But this circuit cannot be fully calculated by analytical methods or numerical simulations. As there is no prototype of the extraction kicker modules in the BRing, a simplified analytical model for the kicker impedance with windowframe magnet is used in the estimates of the transverse kicker impedance [11,12]. The impedance is given by

$$
Z_{1}^{\perp}=\frac{c \omega \mu_{0}^{2} L^{2}}{4 a^{2} Z_{k}}
$$

where $c$ is the speed of light, $\mu_{0}$ is the permeability of vacuum, $L$ is the length, $2 a$ is the gap of the kicker magnet, $Z_{k}=-i \omega \mathcal{L}+Z_{g}$ with $\mathcal{L}$ the inductance of the windings and $Z_{g}$ the impedances of the generator and the cable is the total impedance of the circuit.

After calculating the transverse impedances of the extraction kicker modules by the Eq. (3), the transverse kicker impedance in the BRing is given by

$$
Z_{1, \mathrm{kicker}}^{\perp}(\omega)=\frac{2.6 \times 10^{8} \omega+i 58 \omega^{2}}{\omega^{2}+2.0 \times 10^{13}}(\mathrm{k} \Omega / \mathrm{m})
$$

TABLE II. The main parameters of one extraction kicker module in the BRing.

\begin{tabular}{lc}
\hline \hline Parameter & Value \\
\hline Length $L$ & $558 \mathrm{~mm}$ \\
Gap $2 a$ & $80 \mathrm{~mm}$ \\
Inductance $\mathcal{L}$ & $2.8 \mu \mathrm{H}$ \\
Impedance $Z_{g}$ & $12.5 \Omega$ \\
\hline \hline
\end{tabular}

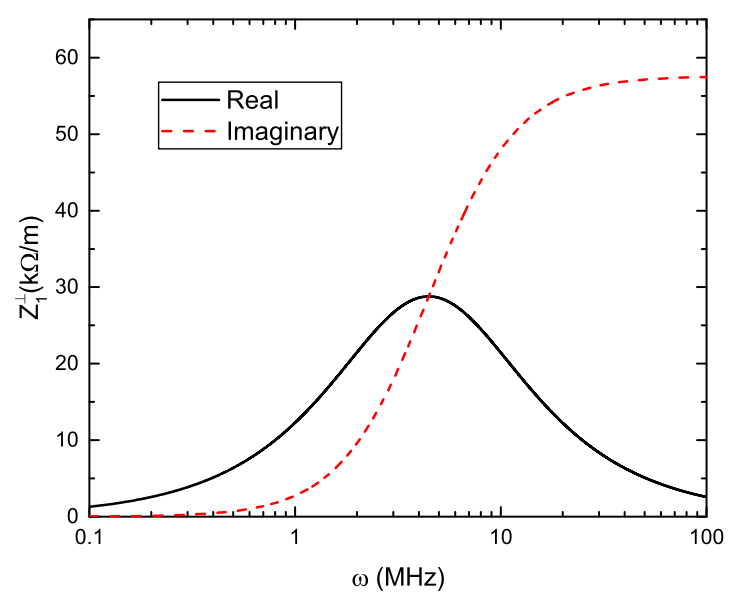

FIG. 2. The transverse kicker impedance changes over angular frequency.

and shown in the Fig. 2. Unlike the transverse resistive wall impedance, the resistive part of the transverse kicker impedance tends toward zero at the low angular frequency and it has the maximal value at the frequency around $\omega=4.5 \mathrm{MHz}$.

\section{Transverse broadband impedances}

Transverse broadband impedances in the BRing come from holes, steps, bellows, collimators, etc. The holes on the vacuum chamber are mainly introduced by the vacuum pumps whose port radii are 75 or $100 \mathrm{~mm}$. The $75-\mathrm{mm}$ radius pumps are $123 \mathrm{NEG}$ (non-evaporable getter) pumps, 92 SIPs (sputter ion pump) and 18 TMPs (turbo molecular pump), and the 100-mm-radius pumps are 44 TSPs (titanium sublimation pump). The total transverse impedance from these sources in the BRing is about $-i 30 \mathrm{k} \Omega / \mathrm{m}$. Another transverse impedance from the vacuum system is the transverse step impedance. After considering all pipe transitions, the total transverse step impedance is about $-i 55 \mathrm{k} \Omega / \mathrm{m}$. Bellows in the vacuum system also introduce a transverse broadband impedance. In the BRing, there are 135 bellows, which gives the total transverse impedance $-i 32 \mathrm{k} \Omega / \mathrm{m}$.

But for collimator system, the transverse impedance cannot be calculated by the analytical method. The Wakefield solver in the CST Studio Suite has been used to simulate it [13]. Half of the 3D model is shown in the Fig. 3. The beam path is shifted a distance (several different values in simulations) from the central orbit to get the transverse impedance [14]. All simulations give almost identical transverse impedance (around $-i 1.6 \mathrm{k} \Omega / \mathrm{m}$ ) when the frequency is larger than the $1 \mathrm{MHz}$. Simulation results are shown in the Fig. 4. The transverse impedance changes a lot when the frequency is low because the accuracy of wake-to-impedance transform at the low frequency is not as good as it is at the high frequency due to the FFT algorithm issues. As there are 27 collimators in the BRing and each 


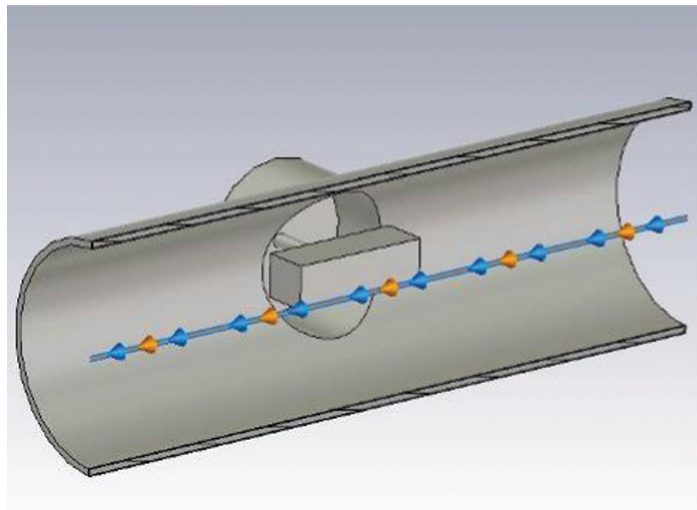

FIG. 3. Half of the 3D collimator model used in the simulation.

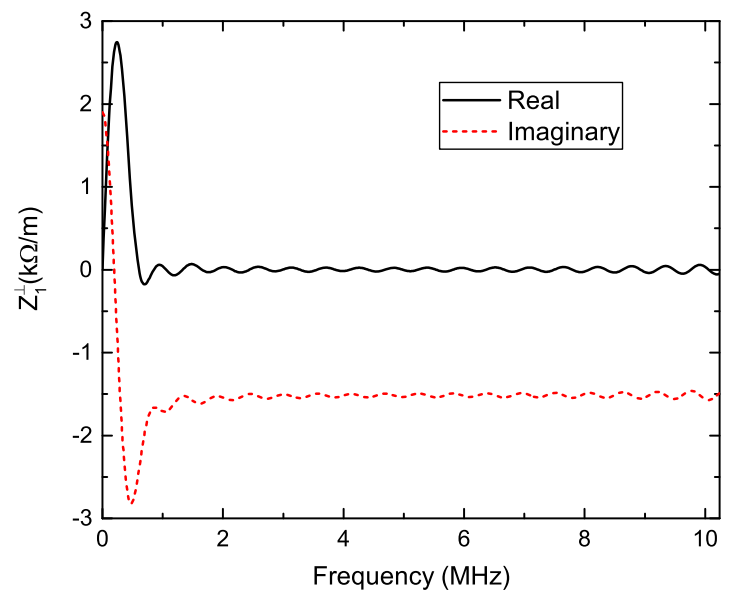

FIG. 4. The transverse impedance in a simulation (the beam path shift is $20 \mathrm{~mm}$ ).

collimator has other holes on the vacuum chamber, the total transverse collimator impedance is $-i 44 \mathrm{k} \Omega / \mathrm{m}$. Then, the whole transverse broadband impedance in the BRing is about $-i 161 \mathrm{k} \Omega / \mathrm{m}$.

\section{A version of the BRing transverse impedance model}

Leaving out some beam diagnostics instruments which have been optimized for reducing the transverse impedance, a BRing transverse impedance model can be given by

$$
\begin{aligned}
Z_{1}^{H}(\omega)= & \frac{1.9 \times 10^{5}}{\sqrt{|\omega|}}(\operatorname{sign}(\omega)-i) \\
& +\frac{2.6 \times 10^{8} \omega+i 58 \omega^{2}}{\omega^{2}+2.0 \times 10^{13}}-i 161(\mathrm{k} \Omega / \mathrm{m}), \\
Z_{1}^{V}(\omega)= & \frac{5.6 \times 10^{5}}{\sqrt{|\omega|}}(\operatorname{sign}(\omega)-i) \\
& +\frac{2.6 \times 10^{8} \omega+i 58 \omega^{2}}{\omega^{2}+2.0 \times 10^{13}}-i 161(\mathrm{k} \Omega / \mathrm{m}),
\end{aligned}
$$

where $Z_{1}^{H}(\omega)$ is the horizontal impedance and $Z_{1}^{V}(\omega)$ is the vertical impedance. This model is a first and preliminary estimate of the total transverse impedance of the BRing, in which only the transverse resistive wall impedance has considered the difference between the horizontal and vertical direction. But the dominant impedance sources have been included in this first version transverse impedance model now, which provides bases for more accurate analysis.

\section{TRANSVERSE COLLECTIVE INSTABILITIES IN THE BRING}

Transverse instabilities in the synchrotrons mainly include mode-coupling instability, coupled-bunch instability, head-tail instability and unbunched beam instability. These transverse instabilities have different corresponding sources (mainly different impedances) and only exist under specific conditions. Sources and conditions for four transverse instabilities are listed in the Table III [5].

In the BRing, transverse head-tail instability will not occur because the chromaticity will be corrected to zero in the heavy ion mode and proton mode. This instability is not included in this paper. And due to this, all analysis or simulations in the paper are with the zero chromaticity. For transverse mode-coupling instability and transverse coupled-bunch instability, threshold and growth times or growth rates are calculated by analytical methods and CISP simulations in all related manipulations with three reference beams in the Table I. For transverse unbunched beam instability, it is very difficult to calculate the growth rates with the special transverse distribution in the BRing. Only simulation results are given for this instability. Besides, some more detailed simulations have been performed in several conditions to test whether the instability modes are the specific modes predicted by the analytical models, or to study the situations that cannot be described in the analytical models. In additional, because the betatron frequency spread from chromaticity, momentum spread or nonlinear magnets is very small in the current design and the spread from the space charge usually cannot cover the coherent frequency due to the large incoherent tune shift, all analytical and simulation results of transverse instabilities do not include the Landau damping. From all analysis made, the analytical results and the simulation results agree with each other and both predict that the BRing may be influenced by all three kinds of transverse instabilities.

\section{A. Transverse mode-coupling instability}

As the beam intensity increases, the shift of each longitudinal azimuthal mode becomes so large that two adjacent modes may overlap each other, which leads to the transverse mode-coupling instability. In the two-macroparticle model, the even modes will be shifted downward 
TABLE III. Sources and conditions for different transverse instabilities.

\begin{tabular}{lll}
\hline \hline Transverse instability & \multicolumn{1}{c}{ Conditions } & \multicolumn{1}{c}{ Sources } \\
\hline Mode-coupling instability & $\begin{array}{l}\text { 1. Bunched beam } \\
\text { 2. Beam intensity or transverse } \\
\text { impedance is larger than the threshold } \\
\text { 3. Chromaticity is very close to 0 }\end{array}$ & 1. Wake field exits in the bunch length \\
Coupled-bunch instability & $\begin{array}{l}\text { 1. Multi-bunched beam } \\
\text { 1. Bunched beam } \\
\text { Head-tail instability }\end{array}$ & $\begin{array}{l}\text { 2. Chromaticity is not zero } \\
\text { 1. Unbunched beam }\end{array}$ \\
Unbunched beam instability field exits among the bunches \\
\end{tabular}

and the odd modes will be shifted upward. When the beam current is very large, even and odd modes will collide and merge with each other, resulting in two conjugated complex eigenfrequencies, thus introducing instability. This is also true for the bunch with a distribution, like air-bag distribution, in which the $m=0$ mode $\nu_{\beta}$ and the $m=-1$ mode $\nu_{\beta}-\nu_{s}$ will collide. All analytical models confirm that this instability has an intensity threshold. An approximate threshold for general transverse impedances is given by [5]

$$
\Upsilon=\left|\frac{\left.Z e I_{b} \bar{\beta}_{\perp} Z_{1}^{\perp}\right|_{\mathrm{eff}} ^{m}}{2 \beta E_{0} \omega_{s} \tau_{L}}\right| \leq 1,
$$

with Sacherer's sinusoidal modes and assuming the frequency shift is about $\omega_{s}$, where

$$
\begin{gathered}
\left.Z_{1}^{\perp}\right|_{\mathrm{eff}} ^{m}=\frac{\int d \omega\left[\beta_{\perp} Z_{1}^{\perp}(\omega)\right] h_{m}(\omega)}{\bar{\beta}_{\perp} \int d \omega h_{m}(\omega)}, \\
h_{m}(\omega) \approx \frac{4(m+1)^{2}}{\pi^{2}} \frac{1+(-1)^{m} \cos \pi y}{\left[y^{2}-(m+1)^{2}\right]^{2}},
\end{gathered}
$$

$Z e$ is the charge of the particle, $I_{b}=Z e N_{b} / T_{0}$ is the average bunch current, $N_{b}$ is the number of particles in the bunch, $T_{0}$ is the revolution period, $\bar{\beta}_{\perp}$ is the average betatron function, $\beta_{\perp} Z_{1}^{\perp}(\omega) / \bar{\beta}_{\perp}$ is the transverse impedance with the local betatron function used as a weight, $h_{m}(\omega)$ is the power spectra, $y=\omega \tau_{L} / \pi, \tau_{L}$ is the total length of the bunch in time, $\beta=v / c, v$ is the velocity of the reference particle, $E_{0}$ is the total energy of the particle and $\omega_{s}$ is the synchrotron angular frequency.

In the BRing, the amplitude of the rf voltage $V_{r f}$ and the related bunch length $\tau_{L}$ are listed in the Table IV. The total bunch length is scaled with revolution time $T_{0}$. Applying the transverse impedance model and the parameters in the Table I, along with the parameters in the Table IV, into the Eq. (7), the thresholds in the different manipulations (marked by M1, M2, M3, and M4) are calculated and shown in the Table V. Except in the M4(p), the values of $\Upsilon_{x}$ and $\Upsilon_{y}$ are far from the thresholds, which means the transverse mode-coupling instability may only influence the proton beam in the second acceleration. The threshold

\begin{tabular}{|c|c|c|}
\hline Manipulation & rf voltage $V_{r f}$ & Bunch length $\tau_{L}$ \\
\hline $\begin{array}{l}\text { M1: Capturing in the first acceleration } \\
\text { at the injection energy }\end{array}$ & $\begin{array}{c}8.21 \mathrm{kV}\left(\mathrm{U}^{34+}\right) \\
15.59 \mathrm{kV}\left(\mathrm{Kr}^{19+}\right) \\
5.86 \mathrm{kV}(\mathrm{p})\end{array}$ & $\begin{array}{l}\text { About } 0.33 T_{0}\left(\mathrm{U}^{34+}\right) \\
\text { About } 0.33 T_{0}\left(\mathrm{Kr}^{19+}\right) \\
\quad \text { About } 0.5 T_{0}(\mathrm{p})\end{array}$ \\
\hline $\begin{array}{l}\text { M2: Accelerating in the first acceleration } \\
\text { at the middle energy }\end{array}$ & $\begin{array}{c}240 \mathrm{kV}\left(\mathrm{U}^{34+}\right) \\
232.6 \mathrm{kV}\left(\mathrm{Kr}^{19+}\right) \\
194 \mathrm{kV}(\mathrm{p})\end{array}$ & $\begin{array}{c}0.22 T_{0}\left(\mathrm{U}^{34+}\right) \\
0.09 T_{0}\left(\mathrm{Kr}^{19+}\right) \\
0.11 T_{0}(\mathrm{p})\end{array}$ \\
\hline $\begin{array}{l}\text { M3: Capturing voltage in the second } \\
\text { acceleration at the middle energy }\end{array}$ & $\begin{array}{l}5.14 \mathrm{kV}\left(\mathrm{U}^{34+}\right) \\
6.47 \mathrm{kV}\left(\mathrm{Kr}^{19+}\right) \\
2.37 \mathrm{kV}(\mathrm{p})\end{array}$ & $\begin{array}{l}\text { About } T_{0}\left(\mathrm{U}^{34+}\right) \\
\text { About } T_{0}\left(\mathrm{Kr}^{19+}\right) \\
\quad \text { About } T_{0}(\mathrm{p})\end{array}$ \\
\hline $\begin{array}{l}\text { M4: Accelerating in the second acceleration } \\
\text { at the extraction energy }\end{array}$ & $\begin{array}{c}31.57 \mathrm{kV}\left(\mathrm{U}^{34+}\right) \\
30.75 \mathrm{kV}\left(\mathrm{Kr}^{19+}\right) \\
22.46 \mathrm{kV}(\mathrm{p})\end{array}$ & $\begin{array}{c}0.19 T_{0}\left(\mathrm{U}^{34+}\right) \\
0.23 T_{0}\left(\mathrm{Kr}^{19+}\right) \\
0.26 T_{0}(\mathrm{p})\end{array}$ \\
\hline
\end{tabular}

TABLE IV. The amplitude of the rf voltage and the related bunch length in the different manipulations with three reference beams. 
TABLE V. The $\Upsilon$ for each manipulation with three reference beams.

\begin{tabular}{llll}
\hline \hline Beam & Manipulation & $\Upsilon_{x} \leq$ & $\Upsilon_{y} \leq$ \\
\hline $\mathrm{U}^{34+}$ & M1 & 0.005 & 0.012 \\
& M2 & 0.003 & 0.005 \\
& M3 & 0.033 & 0.072 \\
& M4 & 0.066 & 0.13 \\
$\mathrm{Kr}^{19+}$ & M1 & 0.009 & 0.022 \\
& M2 & 0.013 & 0.028 \\
& M3 & 0.081 & 0.18 \\
$\mathrm{p}$ & M4 & 0.18 & 0.35 \\
& M1 & 0.012 & 0.038 \\
& M2 & 0.018 & 0.043 \\
& M3 & 0.097 & 0.22 \\
\hline \hline
\end{tabular}

predicted in the M4(p) is $1.07 \times 10^{12} \mathrm{ppp}$ which is about half of the required intensity.

To test the worst situation, CISP simulations have been performed under the M4(p) condition to get the shifts of the modes and the intensity threshold. In the simulation, a proton beam at energy $9300 \mathrm{MeV} / \mathrm{u}$, containing $1 \times 10^{4}$ macro-particles, is injected into the ring after matching with the lattice parameters in the Table I and the rf bucket for the M4(p) in the Table IV. The initial distribution in both transverse phase spaces is uniform distribution with the total emittance $5.96 / 2.98 \pi \mathrm{mm}$ mrad and the initial longitudinal phase space distribution is Gaussian distribution with the rms bunch length $\tau_{L} / 6$, where $\tau_{L}$ is in the M4(p). In the whole simulation process, the linear turn-by-turn transformation and the nonlinear rf are always on and time-independent. The bunch synchrotron energy is also unchanged. The wake given by Eqs. (5) and (6) is only calculated within the total length of the bunch. In order to speed up wake interaction calculation, the bunch is sliced into several bins, whose length are $C / 100$ with $C$ the circumference of the ring, so that the wake interaction can be calculated between these bins instead of macroparticles.

After increasing the bunch intensity from $2 \times 10^{7} \mathrm{ppp}$ to $2 \times 10^{12}$ ppp in 51 simulations, the spectra given by the simulations are shown in the Fig. 5. Because the synchrotron tune is about $1.08 \times 10^{-5}$ while the minimal frequency interval in the FFT for $5.2 \times 10^{5}$-turns data is larger than $1.91 \times 10^{-6}$, the spectrum is a little blurred. But in the spectrum, the $m=0$ mode can still be recognized to shift downward. Same with the analytical prediction, the instability occurs firstly in the vertical direction and it is possible to identify the $m=-1$ mode in this direction near the collision point. The boundary between the blue region and the red region gives the instability threshold $6.2 \times 10^{11} \mathrm{ppp}$. The threshold in the simulation is less than the one in the analytical method, which results from the fact that the instability actually begins when the frequency shift is less

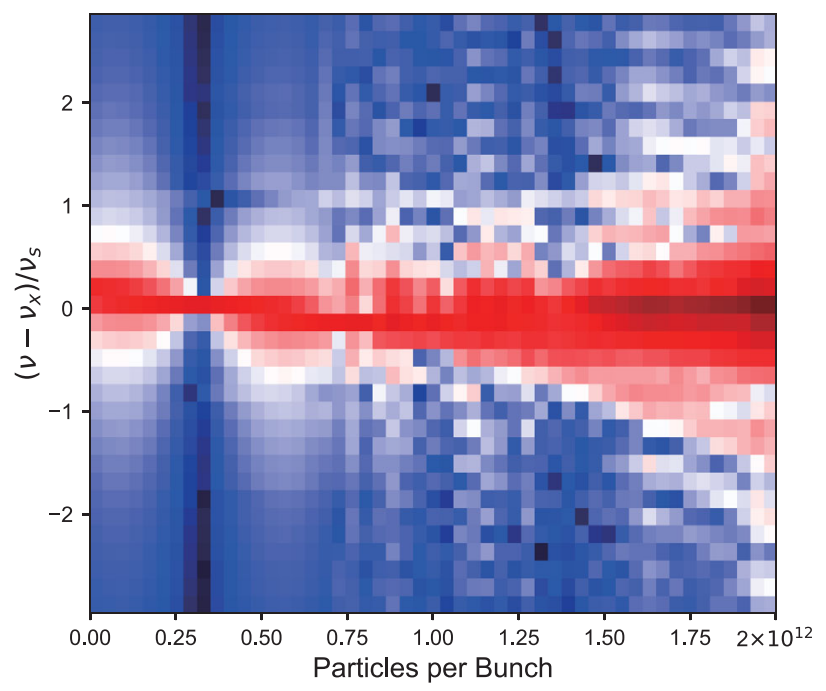

(a) Spectrum for the horizontal direction.

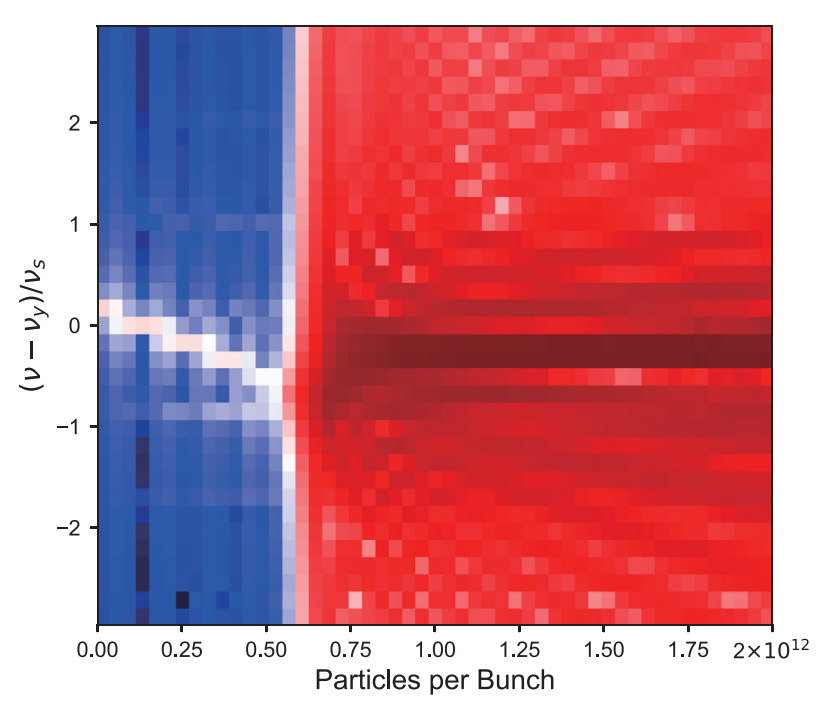

(b) Spectrum for the vertical direction.

FIG. 5. The intensity is changed with a chosen step from a very small value $2 \times 10^{7}$ ppp to $2 \times 10^{12}$ ppp in 51 simulations. FFT algorithm is used to get the spectrum from the bunch displacement in every simulation.

than the $\omega_{s}$. The analytical model in the Eq. (7) will always underestimate the instability. Besides, the longitudinal distribution used in the simulations is similar to a Gaussian distribution with the rms bunch length $\tau_{L} / 6$ which is true in the BRing. It is different from the distribution in the Sacherer's sinusoidal modes.

The presence of the transverse mode-coupling instability is not equal to the conclusion that this instability can lead to a serious consequence. The growth time of the instability should be compared with the duration time of the corresponding manipulation. The simulation can give information about that. By fitting the envelope of the vertical bunch displacement, the growth time in the simulation is around $34 \mathrm{~ms}$. The kinetic energy and the length of the bunch will 
change a lot in this time, thus this instability can only have a little impact on the beam. And the transverse mode-coupling instability can be cured with the chromaticity easily. There is no need to add specific instruments like a transverse feedback system into the BRing to cure this instability.

\section{B. Transverse coupled-bunch instability}

When there are several bunches in the synchrotron, the motion of these bunches can be coupled by the wake field longer than the bunch spacing, which makes the displacements of the bunches increase over time and leads to the beam loss. This is called the transverse coupled-bunch instability. If $M$ identical equally-spaced bunches are in the ring, $M$ transverse coupled modes, characterized by $\mu=0, \ldots, M-1$, will be stimulated. With the Sacherer's sinusoidal modes, the growth times or rates of different coupled-bunch modes are given by [5]

$$
\begin{aligned}
\frac{1}{\tau_{m \mu}} \approx & -\frac{1}{1+m} \frac{Z e M I_{b} \omega_{0}}{4 \pi \beta E_{0}} \\
& \times \frac{\sum_{q} \operatorname{Re}\left[\left.\beta_{\perp} Z_{1}^{\perp}\right|_{R W}\left(\omega_{q}\right)\right] h_{m}\left(\omega_{q}-\chi / \tau_{L}\right)}{B \sum_{q} h_{m}\left(\omega_{q}-\chi / \tau_{L}\right)},
\end{aligned}
$$

where $\omega_{q}=(q M+\mu) \omega_{0}+\omega_{\beta}+m \omega_{s}, q=0, \pm 1, \ldots, \omega_{0}$ is the revolution angular frequency, $\omega_{\beta}$ is the betatron angular frequency, $\omega_{s}$ is the synchrotron angular frequency, $I_{b}$ is the average current per bunch, $B=M \tau_{L} / T_{0}, \chi=\omega_{\xi} \tau_{L}$ is the chromaticity phase shift and $\omega_{\xi}=\xi \omega_{0} / \eta$ is the betatron angular frequency shift due to chromaticity in which $\xi$ is the chromaticity and $\eta$ is the slip factor. However, only the dominant radial mode $k=0$ in each azimuthal mode $m$ is included in the Eq. (10). The most serious transverse coupled-bunch instability that occurs in nearly all accelerator rings is the one driven by the resistive wall [5].

In the BRing, there are 3 heavy ion bunches or 2 proton bunches in the first acceleration. The transverse coupled-bunch instability may be stimulated by the transverse resistive wall impedance. With the transverse resistive wall model given by the Eqs. (1), (2) and the parameters in the Tables I, IV, the growth times of the strongest modes are calculated by the Eq. (10) and listed in the Table VI. In the heavy ion mode, the strongest horizontal mode [marked by $(q, m, \mu)]$ is the $(-3,0,0)$ mode while the strongest vertical mode is the $(-3,0,1)$ mode. And in the proton mode, the strongest horizontal mode is the $(-6,0,0)$ mode while the strongest vertical mode is the $(-5,0,1)$ mode. They are all related to the frequency $\omega=-(1-[Q]) \omega_{0}$ which is known as the $(1-Q)$ line. From the results in the Table VI, all the growth times in the vertical direction are shorter than the duration times of the first acceleration, which means the vertical coupled-bunch instability will influence the beam in the BRing. In the horizontal direction, only the growth times in the $\mathrm{Kr}^{19+}$ beam are a little shorter than the duration time. Especially, the vertical growth times in the $\mathrm{Kr}^{19+}$ beam are much shorter than the duration time and the instability will be very serious, which has been tested specifically in the detailed simulation.

CISP simulations with the time-independent parameters have been performed. In each simulation, the number of bunches is the same with analytical models given above. Each bunch contains $1 \times 10^{4}$ macroparticles. Before the turn-by-turn simulation process, the bunch is matched with the lattice parameters in the Table I and the rf bucket for the M1 and M2 in the Table IV. Similar to the situation in the transverse mode-coupling instability, the linear turn-byturn transformation and the nonlinear rf are always on and time-independent. The bunch is also sliced into several bins which have the length $C / 100$. The initial distribution of the bunches is the same, just with different emittance and bunch length. But it is very different that the wake is truncated after 3 turns in this instability. The growth times can be calculated by fitting the envelope of the displacement of one bunch. They are listed and compared with the analytical results in the Table VI. The growth times from simulations are always slightly less than the analytical results. This phenomenon may result from the fact that the analytical model just gives the growth time of the strongest mode while there are many other unstable radial modes or azimuthal modes. But each simulation result includes all these modes. Besides, the realistic longitudinal distribution is slightly different from the distribution used in the Sacherer's sinusoidal modes. On the other hand, the number of turns, which determines when the wake is truncated, may influence the simulation results. In the

\begin{tabular}{|c|c|c|c|c|c|c|}
\hline & & \multicolumn{2}{|c|}{ Horizontal } & \multicolumn{2}{|c|}{ Vertical } & \multirow[b]{2}{*}{ Duration time } \\
\hline \multicolumn{2}{|c|}{ Particle and manipulation } & Analytical & Simulation & Analytical & Simulation & \\
\hline \multirow[t]{2}{*}{$\mathrm{U}^{34+}$} & M1 & $193 \mathrm{~ms}$ & $140 \mathrm{~ms}$ & $59 \mathrm{~ms}$ & $46 \mathrm{~ms}$ & \multirow[t]{2}{*}{$76 \mathrm{~ms}$} \\
\hline & M2 & $136 \mathrm{~ms}$ & $102 \mathrm{~ms}$ & $42 \mathrm{~ms}$ & $32 \mathrm{~ms}$ & \\
\hline \multirow[t]{2}{*}{$\mathrm{Kr}^{19+}$} & M1 & $60 \mathrm{~ms}$ & $43 \mathrm{~ms}$ & $18 \mathrm{~ms}$ & $14 \mathrm{~ms}$ & \multirow[t]{2}{*}{$68 \mathrm{~ms}$} \\
\hline & M2 & $51 \mathrm{~ms}$ & $36 \mathrm{~ms}$ & $16 \mathrm{~ms}$ & $12 \mathrm{~ms}$ & \\
\hline \multirow[t]{2}{*}{$\mathrm{p}$} & M1 & $65 \mathrm{~ms}$ & $47 \mathrm{~ms}$ & $17 \mathrm{~ms}$ & $14 \mathrm{~ms}$ & \multirow[t]{2}{*}{$33 \mathrm{~ms}$} \\
\hline & M2 & $81 \mathrm{~ms}$ & $57 \mathrm{~ms}$ & $21 \mathrm{~ms}$ & $16 \mathrm{~ms}$ & \\
\hline
\end{tabular}

TABLE VI. Growth times of the transverse coupled-bunch instability in the M1 and M2. 
same $30 \mathrm{MeV} / \mathrm{u} \mathrm{Kr}^{19+}$ simulation, when the wake is truncated after 14 turns, the result is about $17 \mathrm{~ms}$ instead of $14 \mathrm{~ms}$ in the Table VI.

However, in reality, the parameters of the rf are timedependent, and the synchrotron energy of the beam keeps changing in the first acceleration. Besides, the longitudinal distribution of the beam is not an exact Gaussian distribution due to the capturing process in the BRing. So, when the $\mathrm{rf}$ is set to read the amplitude voltage and the synchrotron phase from two data files while other parameters are the same with the previous situations, CISP simulations in the whole first acceleration are performed to further ensure that the BRing may be affected by this instability in the vertical direction as described in the Table VI. First, the worst situation, i.e., the first acceleration for the $\mathrm{Kr}^{19+}$ beam, is shown in the Figs. 6 and 7. There is a beam loss due to the transverse coupled-bunch instability at the beginning of the debunching stage. The vertical normalized emittance increases in the whole first acceleration. The beam loss and the emittance growth have confirmed that in the realistic condition, the instability will actually limit the intensity and the beam quality of the BRing. The coordinates of particles in three turns have been exported to analyze the betatron phase advance between two adjacent bunches. By fitting the displacements of the bunches with a sine function, which is shown in the Fig. 8, the phase advance is around $0.366 \pi$ in the horizontal direction and $0.380 \pi$ in the vertical direction. These phase advances are identical with the ones, $0.367 \pi$ in the horizontal direction and $0.380 \pi$ in the vertical direction, in the analytical model, which also confirms the consistency of both methods again [15]. The transverse coupledbunch instability will influence the quality of the $\mathrm{U}^{34+}$ and proton beam in the vertical direction, as shown in the

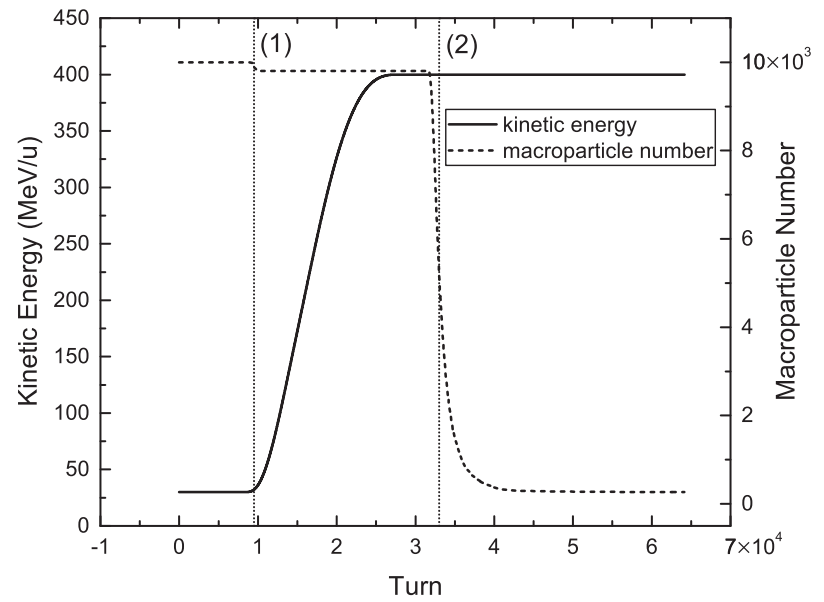

FIG. 6. Beam loss and kinetic energy change over time in the $\mathrm{Kr}^{19+}$ simulation. Beam loss (1) is related to the longitudinal dynamics and the beam loss (2) is related to the transverse coupled-bunch instability.

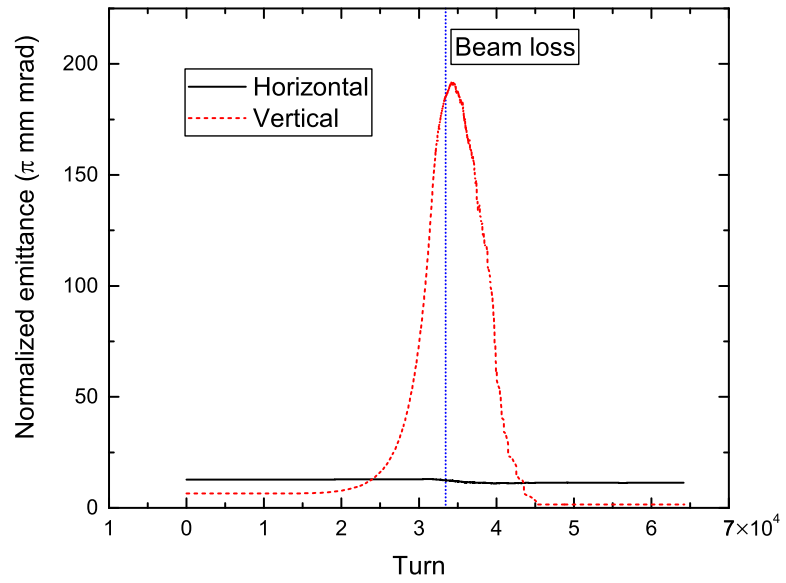

FIG. 7. Normalized emittance in both directions changes over time in the $\mathrm{Kr}^{19+}$ simulation. The vertical normalized emittance increases before the beam loss while the horizontal normalized emittance changes a little.

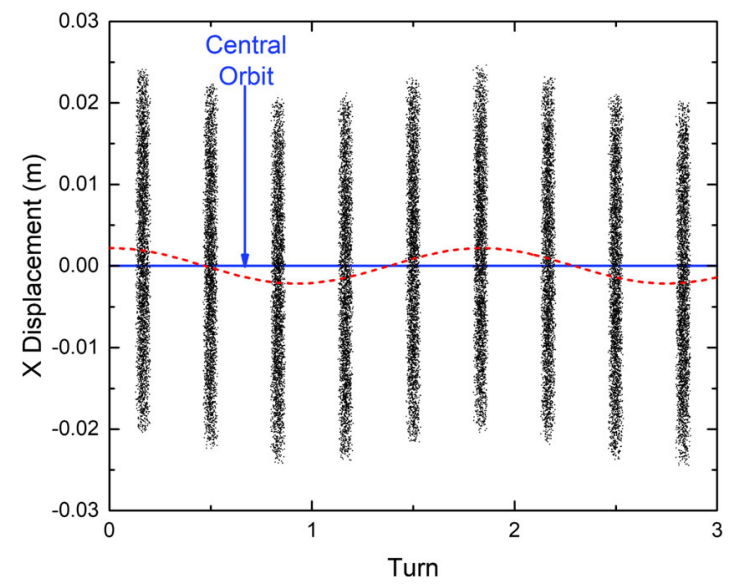

(a) The horizontal displacements of the bunches are fitted to the dashed line.

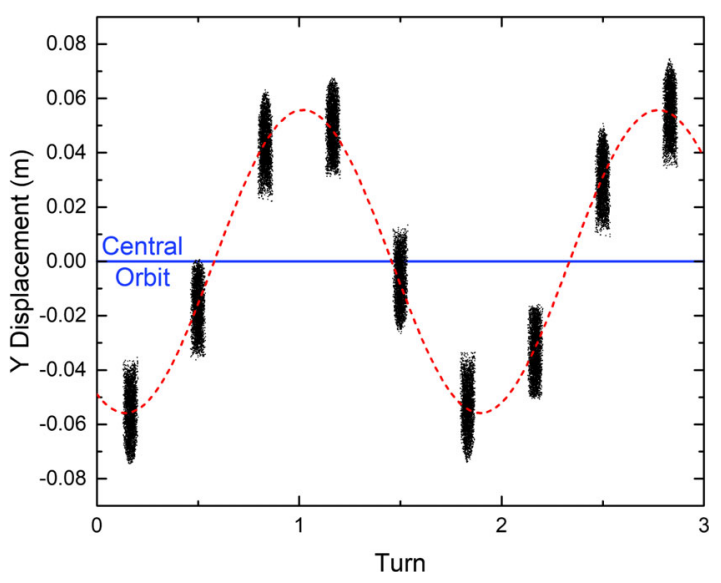

(b) The vertical displacements of the bunches are fitted to the dashed line.

FIG. 8. Bunches oscillate around the central orbit and their displacements can be fitted by a sine function. 


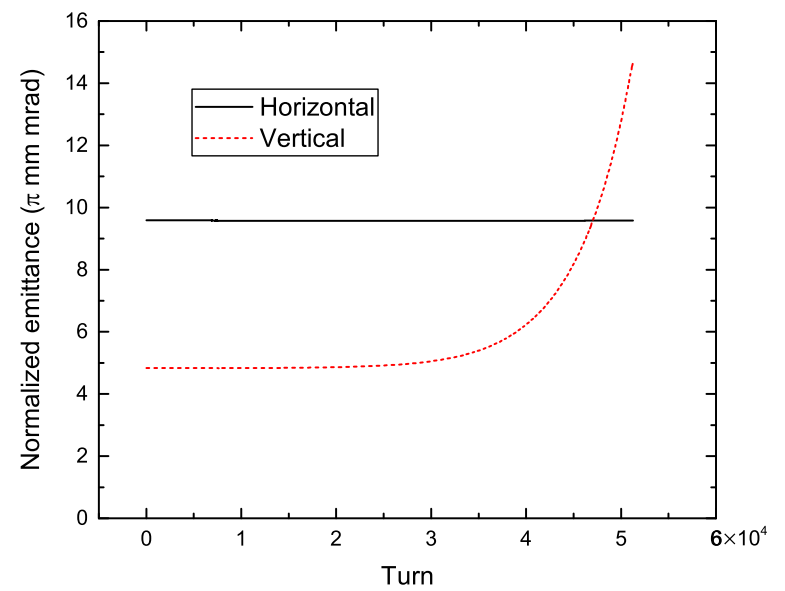

FIG. 9. Normalized emittance in both directions changes over time in the $\mathrm{U}^{34+}$ simulation. The vertical normalized emittance increases a lot while the horizontal normalized emittance changes a little.

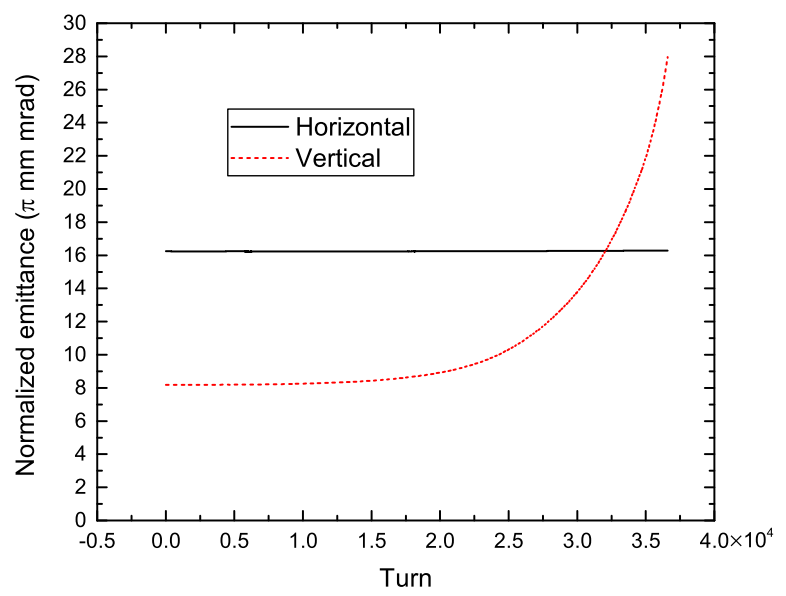

FIG. 10. Normalized emittance in both directions changes over time in the proton simulation. The vertical normalized emittance increases a lot while the horizontal normalized emittance changes a little.

Figs. 9 and 10. This is the same with the results from the Table VI.

According to all analysis, the transverse coupledbunch instability will have serious detrimental impacts on the heavy ion beam in the BRing, like limiting the beam intensity and reducing the beam quality. A transverse feedback system has been proposed to cure this instability.

\section{Transverse unbunched beam instability}

The coasting beam also has a transverse dipole density that depends on the location along the ring and the time, which gives the transverse dipole modes marked by $\nu_{n}=n+\nu_{\beta}$. Landau damping due to the finite momentum spread and chromaticity is a stabilizing mechanism of these
TABLE VII. The main parameters of the coasting beams.

\begin{tabular}{lc}
\hline \hline Manipulation & Momentum spread $\pm 3 \delta_{\mathrm{rms}}$ \\
\hline M5: Injection & $\pm 0.2 \%$ (all species) \\
M6: Middle & $\pm 0.05 \%\left(\mathrm{U}^{34+}\right)$ \\
& $\pm 0.06 \%\left(\mathrm{Kr}^{19+}\right)$ \\
& $\pm 0.09 \%(\mathrm{p})$ \\
M6: Slow extraction & $\pm 0.04 \%\left(\mathrm{U}^{34+}\right)$ \\
& $\pm 0.09 \%\left(\mathrm{Kr}^{19+}\right)$ \\
& $\pm 1 \%(\mathrm{p})$ \\
\hline \hline
\end{tabular}

TABLE VIII. The growth rates of the transverse unbunched beam instability.

\begin{tabular}{lcccr}
\hline \multicolumn{2}{l}{$\begin{array}{l}\text { Particle and } \\
\text { manipulation }\end{array}$} & Horizontal & Vertical & $\begin{array}{c}\text { Duration } \\
\text { time }\end{array}$ \\
\hline $\mathrm{U}^{34+}$ & M5 & $\approx 0 \mathrm{~ms}^{-1}$ & $0.036 \mathrm{~ms}^{-1}$ & $58 \mathrm{~ms}$ \\
& M6 & $0.0069 \mathrm{~ms}^{-1}$ & $0.065 \mathrm{~ms}^{-1}$ & $\approx 0 \mathrm{~ms}$ \\
& M7 & $\approx 0 \mathrm{~ms}^{-1}$ & $0.036 \mathrm{~ms}^{-1}$ & $>100 \mathrm{~ms}$ \\
$\mathrm{Kr}^{19+}$ & M5 & $0.015 \mathrm{~ms}^{-1}$ & $0.10 \mathrm{~ms}^{-1}$ & $58 \mathrm{~ms}$ \\
& M6 & $0.028 \mathrm{~ms}^{-1}$ & $0.16 \mathrm{~ms}^{-1}$ & $\approx 0 \mathrm{~ms}$ \\
& M7 & $0.0064 \mathrm{~ms}^{-1}$ & $0.073 \mathrm{~ms}^{-1}$ & $>100 \mathrm{~ms}$ \\
$\mathrm{p}$ & M5 & $0.030 \mathrm{~ms}^{-1}$ & $0.16 \mathrm{~ms}^{-1}$ & $30 \mathrm{~ms}$ \\
& M6 & $0.021 \mathrm{~ms}^{-1}$ & $0.14 \mathrm{~ms}^{-1}$ & $\approx 0 \mathrm{~ms}$ \\
& M7 & $\approx 0 \mathrm{~ms}^{-1}$ & $0.019 \mathrm{~ms}^{-1}$ & $>100 \mathrm{~ms}$ \\
\hline \hline
\end{tabular}

modes. However, the tune shift from the imaginary part of the transverse impedance or the space charge effect can lead to the loss of Landau damping [7]. Then, the real part of the transverse impedance will lead to damping or instability if the frequency sampled by the impedance is positive or negative.

In the BRing, the transverse resistive wall impedance contributes most to the real part of the transverse impedance and it is sampled by the negative frequency at the

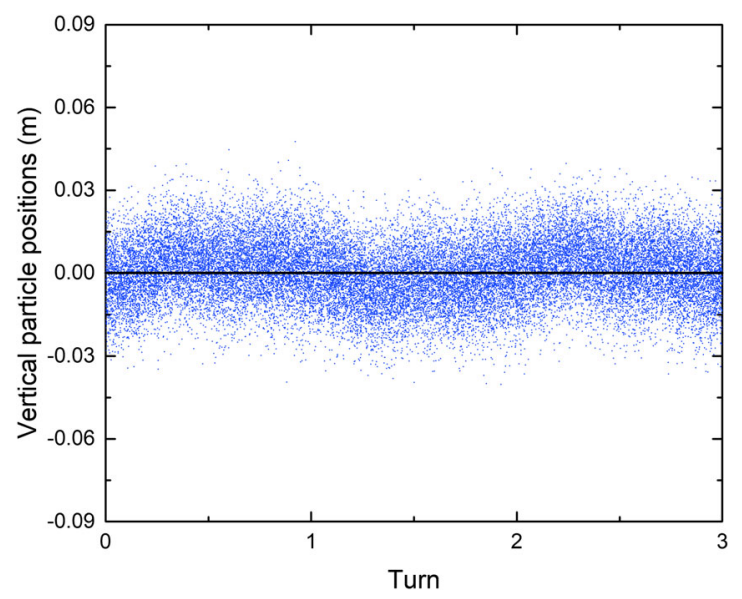

FIG. 11. Coasting beam is oscillating around the central orbit in the $400 \mathrm{MeV} / \mathrm{u} \mathrm{Kr}^{19+}$ beam simulation with the transverse impedance and the space charge. 
$(1-Q)$ line, which may result in the transverse unbunched beam instability. It is very difficult to include the space charge effect in the analytical model for the BRing. Because after the two-plane painting injection, both horizontal phase space distribution and vertical phase space distribution are uniform distributions and almost independent of each other, which results in a very special transverse distribution. Thus, the growth rates of this instability are only calculated by the CISP simulations.

In the simulations, a coasting beam, with Gaussian momentum spread listed in the Table VII and the uniform transverse phase space distribution is injected. It is matched with first linear point-to-point transformation. And there is $1 \times 10^{4}$ macroparticles in this beam. The rf remains off in

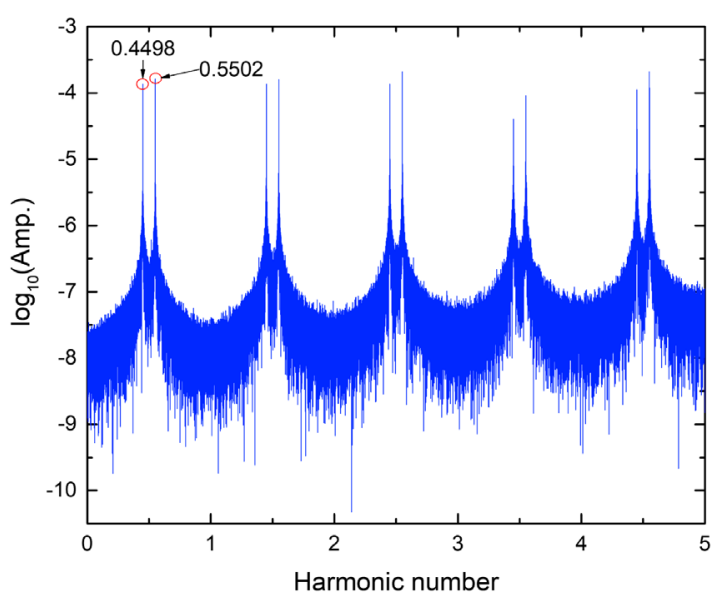

(a) Horizontal betatron sidebands of the first 5 revolution harmonics in the simulation without the transverse impedance but with the space charge.

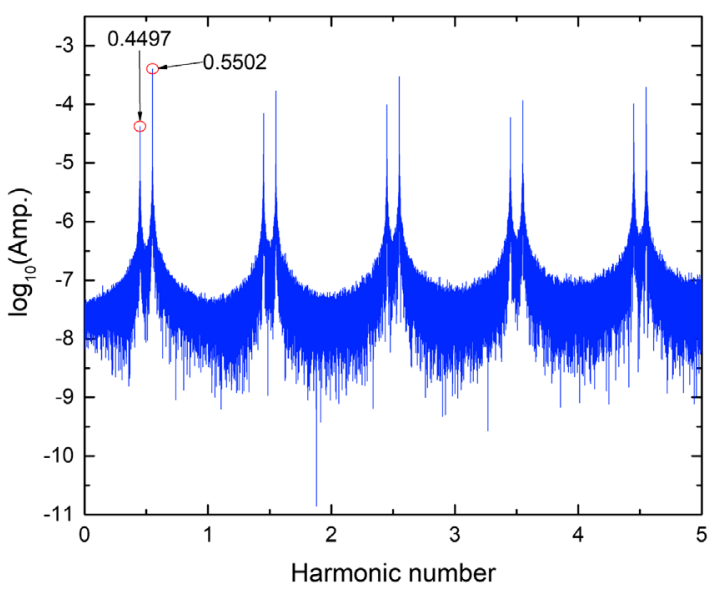

(b) Horizontal betatron sidebands of the first 5 revolution harmonics in the simulation with the transverse impedance and the space charge.

FIG. 12. Betatron sidebands in the horizontal direction. The $(1-Q)$ line is clearly dominant and the lower betatron sidebands grow more than the upper sidebands. the whole simulation process. The space charge force in the beam is calculated at the 100 points along the ring. At each point, the beam is sliced into 100 bins and 2D FFT-PIC is performed in each bin with a rectangular boundary and $64 \times 64$ rectangular grids. While calculating the wake interaction at a fixed point, the beam is also sliced into 100 bins, and the wake is truncated after 3 turns.

After fitting the envelope of the beam displacement, the growth rates of the transverse unbunched beam instability in the different manipulations are shown in the Table VIII. In the M5 and M7, the transverse unbunched beam instability can influence the beam in the BRing, and again the worst situation occurs in the $\mathrm{Kr}^{19+}$ beam. The beam oscillates around the central orbit in the simulation, as

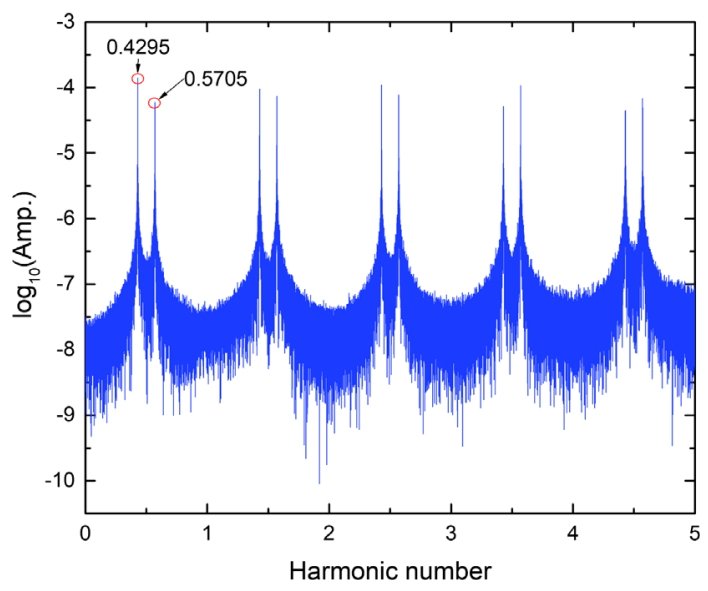

(a) Vertical betatron sidebands of the first 5 revolution harmonics in the simulation without the transverse impedance but with the space charge.

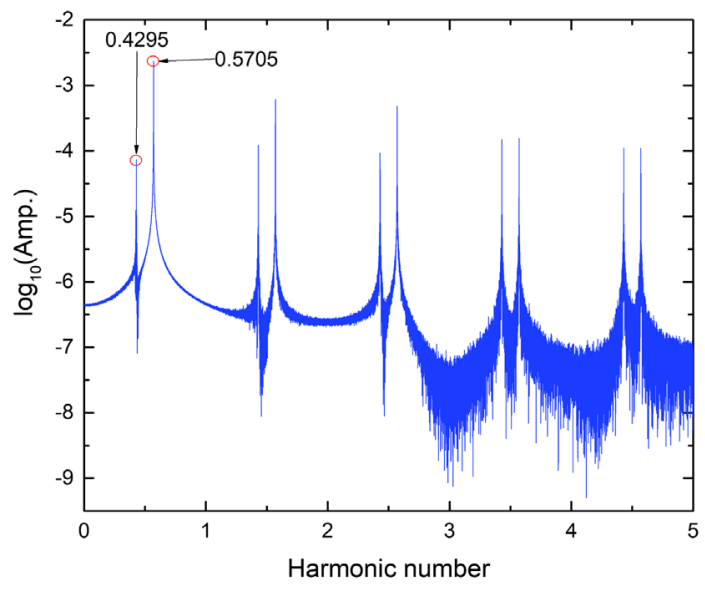

(b) Vertical betatron sidebands of the first 5 revolution harmonics in the simulation with the transverse impedance and the space charge.

FIG. 13. Betatron sidebands in the vertical direction. The $(1-Q)$ line is also clearly dominant and the lower betatron sidebands also grow more than the upper sidebands. 
shown in the Fig. 11, until the beam begins to lose or the bunches are formed by the rf voltage.

To test whether the transverse unbunched beam instability in the simulation is mainly stimulated by the transverse resistive wall impedance at the $(1-Q)$ line, beam central position has been detected at the frequency $\omega=50 \omega_{0}$ and used to perform the FFT. The results are shown in the Figs. 12 and 13 in which the lower betatron sidebands and the upper betatron sidebands are very clear.

In the horizontal direction, the fractional part is set to 0.45 in the simulation. The spectrum generated from the signals gives the upper sidebands 0.4497 and the lower sidebands 0.5502 when the simulation includes the transverse impedance. They are very close to the betatron sidebands expected. And the first lower sideband grows more than the corresponding upper sideband, as a result of the $(1-Q)$ line [5]. In the vertical direction, the phenomenon is similar with the horizontal direction.

From all the results above, the transverse unbunched beam instability will influence the beam in the BRing and the strongest modes are at the $(1-Q)$ line. To cure this instability, the transverse feedback system should cover the lowest frequency which is less than $(1-[Q]) f_{0}$ and the highest frequency which is higher than the loss of Landau damping frequency range [6].

\section{CONCLUSION}

The transverse impedances from the dominant sources in the BRing are all calculated and summarized in this paper, and the full transverse impedance model of the BRing is given for the first time. The transverse impedance model has included the transverse resistive wall impedance, the transverse kicker impedance and several transverse broadband impedances, which has built a framework that can be followed in the future. In these transverse impedances, the transverse resistive wall impedance from the stainless-steel vacuum chamber is the most significant, as almost all the strongest transverse instabilities in the BRing are stimulated by it. In the future, this impedance should be updated if the NEG coating is used in the BRing. The transverse kicker impedance is also important. But from the results shown in the paper, it is not the main source of the transverse instabilities. However, the estimation of the transverse kicker impedance can be very rough only by applying the analytical method. The bench measurement will be used to get the accurate transverse kicker impedance when the prototypes of the kicker modules and their generators are finished in the future. Then, the transverse instability analysis will be updated in order to include the realistic influences introduced by the transverse kicker impedance. It is a long-term task to update all estimation of the transverse impedances.

As to the transverse instabilities, they will influence the maximal intensity and the beam quality of the BRing in almost all manipulations. The transverse coupled-bunch instability will have detrimental impacts on all beams in the first acceleration. This instability is mainly stimulated by the transverse resistive wall impedance and has been proven by the analytical methods and the CISP simulations. There are also transverse unbunched beam instabilities in the injection or the slow extraction of the BRing, which will lead to the distortion or loss in all beams. The CISP simulations have confirmed that this instability is also mainly stimulated by the transverse resistive wall impedance (of course, the more accurate space charge effect should be included in future calculations). The transverse mode-coupling instability is not as serious as previous two instabilities in the BRing. It only occurs in the proton beam whose kinetic energy is close to the extraction energy. And the instability will not affect the beam much because the corresponding manipulation is very short. All the analytical analysis and the CISP simulations that agree with each other have provided two methods to analyze the transverse instabilities in the synchrotron, and given some possible ways, like testing the betatron phase advance between two adjacent bunches or generating the spectra of the displacements, to measure some modes of the transverse instabilities. However, in reality, the closed orbit and the complex geometry of the vacuum chamber can introduce many deviations in the wake interaction and space charge simulations. These will be tested in the further code benchmarks with experiments. After that, whether the numerical models should be improved can be decided.

In the future, with the help of other divisions in the IMP, a transverse feedback system will be added into the BRing to cure the transverse coupled-bunch instability and the transverse unbunched beam instability. And the designs will be simulated by the CISP application to make sure that the transverse instabilities can be damped by a feedback system.

\section{ACKNOWLEDGMENTS}

Thank the support from The National Natural Science Foundation of China (NSFC) (Grant Nos. 11505256 and 11705253) and the support from Guangdong Innovative and Entrepreneurial Research Team Program (No. 2016ZT06G373).

[1] J.C. Yang et al., High intensity heavy ion accelerator facility (HIAF) in China, Nucl. Instrum. Methods Phys. Res., Sect. B 317, 263 (2013).

[2] G. F. Qu, W. P. Chai, J. W. Xia, J. C. Yang, H. Du, Z. S. Li, W. W. Ge, and P. Shang, Two-plane painting injection scheme for BRing of HIAF, Nucl. Sci. Technol. 28, 114 (2017).

[3] D. Y. Yin et al., Generation of short intense heavy-ion pulses in HIAF, Proceedings of IPAC2017, Copenhagen, Denmark (2017). 
[4] A. W. Chao, Physics of Collective Beam Instabilities in High Energy Accelerators (Wiley, New York, 1993).

[5] K. Y. Ng, Physics of Intensity Dependent Beam Instabilities (World Scientific, Singapore, 2006).

[6] U. Blell et al., Transverse damping system at SIS100, Proceedings of EPAC 2006, Edinburgh, Scotland (2006).

[7] V. Kornilov, O. Boine-Frankenheim, and I. Hofmann, Stability of transverse dipole modes in coasting ion beams with nonlinear space charge, octupoles, and chromaticity, Phys. Rev. ST Accel. Beams 11, 014201 (2008).

[8] J. Liu, J. C. Yang, J. W. Xia, D. Y. Yin, G. D. Shen, P. Li, H. Zhao, S. Ruan, and B. Wu, CISP: Simulation platform for collective instabilities in the BRing of HIAF project, Nucl. Instrum. Methods Phys. Res., Sect. A 881, 36 (2018).

[9] J. C. Wang, P. Li, J. C. Yang, Y. J. Yuan, B. Wu, Z. Chai, C. Luo, Z. Q. Dong, W. H. Zheng, H. Zhao, G. W. S. Ruan, J. Liu, X. Chen, K. D. Wang, Z. M. Qin, and B. Yin, Pressure profiles of the BRing based on the simulation used in the CSRm, Nucl. Instrum. Methods Phys. Res., Sect. A 860, 19 (2017).
[10] L. S. Huang, The theoretical and experimental study on the coupling impedance measurement and the collective effects in CSNS/RCS, Ph.D. thesis, University of Chinese Academy of Sciences, 2014.

[11] A. W. Chao, K. H. Mess, M. Tigner, and F. Zimmermann, Handbook of Accelerator Physics and Engineering (World Scientific, Singapore, 1999).

[12] G. Nassibian, The low frequency beam impedance of matched travelling wave kickers, CERN Technical Report No. CERN-PS-84-25-BR, 1984.

[13] CST STUDIO SUITE, https://www.cst.com/.

[14] C. Zannini, L. Rivkin, and G. Rumolo, Electromagnetic simulation of CERN accelerator components and experimental applications, Ph.D. thesis, CERN, 2013.

[15] M. Lonza and H. Schmickler, Proceedings of the CAS-CERN Accelerator School: Intensity Limitations in Particle Beams, Geneva, Switzerland, 2-11 November 2015, edited by W. Herr, CERN Yellow Reports: School Proceedings, Vol. 3, CERN-2017-006-SP (CERN, Geneva, 2017). 